\title{
Actualización Ecocardiográfica en canal atrioventricular disbalanceado derecho
}

\author{
Julio Cesar Biancolini* \\ Fernanda M. Biancolini* \\ Ana M. S. de Dios** \\ Jesus Damsky Barbosa*** \\ Ignacio Lugones $* * * *$
}

* Unidad de Cardiología Infantil, Hospital General de Niños "Dr. Pedro de Elizalde", Buenos Aires, Argentina

** Unidad de Cardiología Infantil, Sanatorio Trinidad Mitre, Buenos Aires, Argentina

***Unidad de Cardiología Infantil, Hospital General de Niños "Dr. Pedro de Elizalde", Buenos Aires, Argentina

**** Unidad de Cirugía Cardiovascular, Hospital General de Niños "Dr. Pedro de Elizalde", Buenos Aires, Argentina

Recibido: 11/02/2021

Aceptado: 28/02/2021

En línea: 30/04/2021

\begin{tabular}{|l|}
\hline Correspondencia \\
\hline Biancolini Julio César \\
jbiancolini@gmail.com
\end{tabular}

Citar como: Biancolini J.m, Biancolini F., De Dios A., Damsky J., Lugones I. Actualización Ecocardiográfica en Canal atrioventricular desbalanceado derecho. Rev Rev Ecocar Pract (RETIC). 2021 (Abril); 4 (1): 37-39. doi: 10.37615/retic.v4n1a1.

Cite this as: Biancolini J.m, Biancolini F., De Dios A., Damsky J., Lugones I. Echocardiographic actualization in disbalanced right atrioventricular canal. Rev Ecocar Pract (RETIC). 2021 (Abril); 4 (1): 37-39. doi: 10.37615/retic.v4n1a1.

\begin{tabular}{l} 
Palabras clave \\
\hline$\triangleright$ Canal atrioventricular \\
$\quad$ disbalanceado \\
$\triangleright$ Defecto del tabique \\
interventricular \\
$\triangleright$ Ecocardiografía \\
$\triangleright$ Corazón univentricular
\end{tabular}

\begin{tabular}{l}
\hline Keywords \\
\hline$\triangleright$ Unbalanced atrioventricular \\
canal defect \\
$\triangleright$ Ventricular septal defect \\
$\triangleright$ Echocardiography \\
$\triangleright$ Functionally univentricular \\
heart \\
\hline
\end{tabular}

\begin{abstract}
RESUMEN
El canal atrioventricular disbalanceado puede ser una patología desafiante al momento de tomar una decisión quirúrgica, especialmente en los pacientes con moderada hipoplasia del ventrículo izquierdo. Es por ello que su abordaje es complejo y deben evaluarse anatómica y hemodinámicamente en forma completa y posiblemente con una modalidad multi-imagen. El presente trabajo brinda una actualización ecocardiográfica para la valoración del canal atrioventricular disbalanceado derecho, con el objetivo de dar al cardiólogo una herramienta para la toma de decisiones quirúrgicas frente a esta compleja patología.
\end{abstract}

\begin{abstract}
Unbalanced atrioventricular canal can be a challenging pathology when making a surgical decision, especially in patients with moderate left ventricular hypoplasia. Therefore, the approach is complex and must be fully evaluated and possibly with a multi-imaging modality. The aim of this paper is present an echocardiographic update for evaluation of right unbalanced atrioventricular septal defect, as a useful tool for cardiologist in making surgical decisions in front of this complex pathology.
\end{abstract}

\section{Presentación}

El canal atrioventricular (CAV) disbalanceado puede ser una patología desafiante al momento de tomar una decisión quirúrgica, especialmente en los pacientes con moderada hipoplasia del ventrículo izquierdo (VI), también llamados disbalanceados "borderline".

Desde hace muchos años está descrito el índice de la válvula atrio-ventricular o "atrioventricular valve index" (AVI) para caracterizar a los pacientes en balanceados y disbalanceados ${ }^{(1)}$. Este índice fue posteriormente modificado (mAVI) y se calcula como el cociente entre el área izquierda de la válvula atrio-ventricular (VAV) y el área total de la misma en una vista subcostal, oblicua anterior izquierda ${ }^{(2)}$.

Esta misma relación se mantiene tomando el diámetro del componente izquierdo y el diámetro total de la VAV en 4 cámaras $^{(3)}$ (Figura 1).
Se define como balanceados a los pacientes con mAVVI entre 0,4 y 0,6, disbalanceados dominantes derechos "borderline" a aquellos cuyo mAVVI se encuentra entre 0,39 y 0,2 y disbalanceados derechos severos a aquellos con mA$\mathrm{VVI}<0.2$. Es decir, si el componente izquierdo representa más de $40 \%$ de la VAV nos encontremos frente a un paciente balanceado, mientras que, si representa menos de un $20 \%$ del total, éste será un caso de disbalance severo.

Nathan et al. ${ }^{(4)}$ utilizaron a la resonancia magnética cardiaca para valorar a esta población y definir la conducta a seguir, definiendo la hipoplasia moderada del ventrículo o de la válvula atrioventricular como volúmenes del VI de 15 a $30 \mathrm{ml} /$ $\mathrm{m}^{2} ; 0,19<\mathrm{AVVI}<0,39$.

Asimismo, se ha descrito en publicaciones previas que los pacientes con una comunicación interventricular (CIV) de gran tamaño presentaban alto índice de mortalidad cuando se los llevaba por un camino biventricular ${ }^{(1)}$. Es correcto suponer que aquellos pacientes con una CIV pequeña poseen un VI que ya se encuentra manejando la mayor parte del volumen minuto sistémico, ya que no existe un cortocircuito de izquierda a derecha demasiado importante 
a través de esa pequeña CIV. Por el contrario, en los pacientes con CIV de gran tamaño, el VI se encuentra desprecargado, es de menor tamaño y su capacidad de mantener el gasto cardíaco una vez realizada la corrección biventricular es incierta.

En 2013, se publicó uno de los estudios multicéntricos más grandes sobre CAV en el cual se evaluó el comportamiento del ángulo que se forma entre ambos extremos de la VAV y la punta del septum interventricular. Se determinó que los pacientes con un ángulo más obtuso eran pacientes más balanceados y aquellos con ángulos más agudos presentaban mayor disbalance (5). Posteriormente, nuestro grupo demostró que en realidad el valor del ángulo es esencialmente una forma indirecta de valorar el tamaño de la CIV asociado al grado de disbalance (3). Los lados de este ángulo en conjunción con la VAV conforman un triángulo cuyo ángulo es el descrito previamente y cuya altura representa el tamaño de la CIV (Figura 1). El valor del ángulo se ve más afectado por el tamaño de la CIV que por el grado de disbalance.

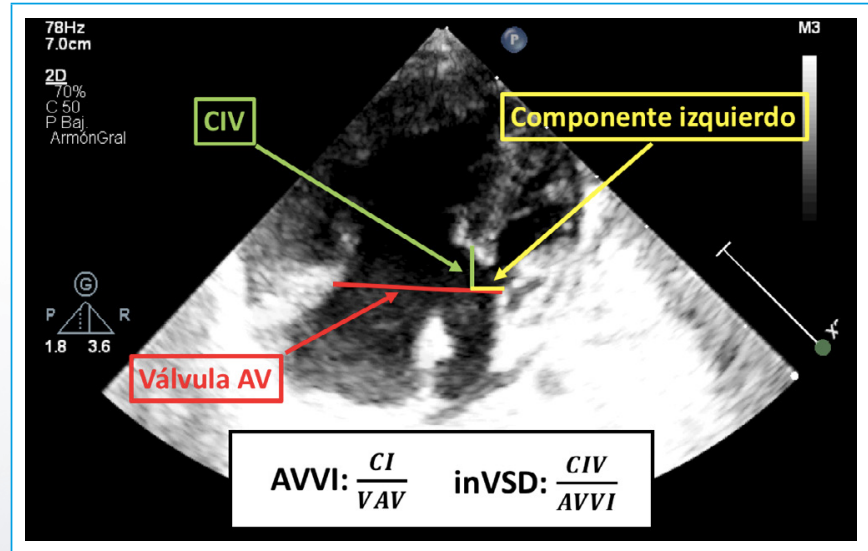

Figura 1. El diámetro total del anillo de la VAV se ve en rojo. El diámetro de la CIV, medido trazando una línea que parte de la punta del septum interventricular y corta el plano de la VAV a 90 grados, se observa en verde. El punto de inserción de las líneas blancas al septum interventricular, representa el ángulo descrito por Cohen. El componente izquierdo de la VAV se extiende desde el punto izquierdo de inserción de la VAV al sitio de intersección con la línea de la CIV, en amarillo. mAVVI: componente izquierdo/diámetro total del anillo de la VAV. inVSD: CIV/diámetro total del anillo de la VAV. Cl: componente izquierdo. VAV: válvula auriculo ventricular. CIV: comunicación interventricular. AVVI auriculo ventricular valve index. inVSD: ventricular septal defect index.

Así, surge la CIV indexada o "indexed ventricular septal defect" (inVSD) como nueva variable ecocardiográfica, y se obtiene realizando el cociente entre el diámetro de la CIV y el diámetro total de la VAV (Figura 1). Es así como dentro de la población con moderado grado de disbalance (mAVVI entre 0.2 y 0.39), los pacientes con valores bajos de inVSD tendrán más posibilidades de superar satisfactoriamente la reparación biventricular que pacientes con valores altos, es decir con CIV demasiado grande, en los que se recomendará una estrategia univentricular o estrategias alternativas (Figura 2).

Aún con la introducción de este nuevo parámetro, la evaluación ecocardiográfica preoperatoria resultaba insuficiente para la toma de decisiones quirúrgicas. Como bien sabemos, en esta cardiopatía congénita las alteraciones de la VAV y su aparato subvalvular son asociaciones frecuentes.

Al analizar la capacidad del VI de manejar el gasto cardíaco luego de la septación, es importante considerar que estas asociaciones pueden modificar "la entrada verdadera" a este ventrículo. Dicho de otra manera, aun logrando un buen diámetro de la mitral durante la reparación biventricular, la presencia de patología subvalvular (mitral en paracaídas, músculos papilares fusionados, músculo papilar único, engrosamiento de las cuerdas tendinosas, presencia de cuerdas accesorias, etc) puede condicionar el ingreso de sangre al VI y afectar su desempeño. Por este motivo, se tornaba imperioso el análisis geométrico de esta región anatómica y su inclusión en el esquema de toma de decisiones quirúrgica.

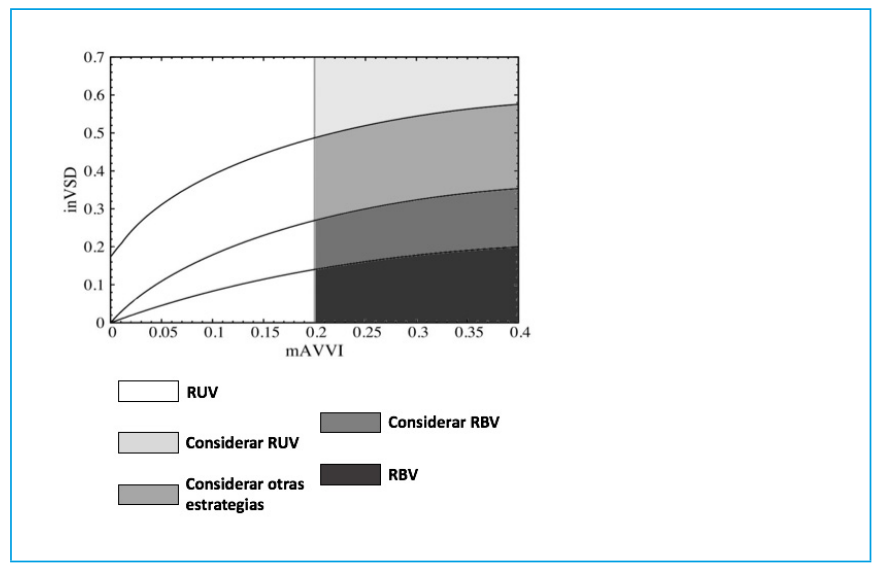

Figura 2. Para la región de $\mathrm{mAVVI}<0.2$, la reparación univentricular se recomienda en virtud del severo disbalance. Para valores de mAVVI entre 0.2 y 0.39 , el valor de la inVSD sugerirá la conducta a seguir. Para valores de inVSD bajo, la reparación biventricular puede ser considerada como estrategia segura, mientras que valores altos de la variable obligan a considerar estrategias alternativas o directamente la paliación univentricular. RUV: reparación univentricular, RBV: reparación biventricular

Szwast y colaboradores describieron el índice de flujo de entrada al ventrículo izquierdo o "left ventricular inflow index" (LVII) (6). Este índice se calcula haciendo el cociente entre la medida del anillo secundario o "secondary annulus" (SA) y el anillo primario izquierdo o "left primary annulus" (LPA). El primero está representado por el menor diámetro de ingreso a la cavidad del VI, medido con Doppler color como una línea extendida entre la cresta del septum interventricular y la pared de VI. Este grupo demostró que los pacientes con un LVII $<0.55$ tenían mortalidad aumentada cuando seguían el camino biventricular. Sin embargo, serias limitaciones se han reportado en su uso(7) y esto es, a nuestro entender, se debe a que esta variable nuevamente se ve influenciada por el tamaño de la CIV (a mayor tamaño del defecto, mayor medida del LPA), no siendo una medida de valoración independiente del tracto de entrada ventricular.

Por estas razones, nuestro grupo está trabajando actualmente en un nuevo índice ecocardiográfico, el anillo secundario indexado o "index secondary anulus" (inSA), que podría tener la capacidad de evaluar la magnitud de la obstrucción subvalvular de manera pura. Se calcula como la relación entre el SA y el diámetro total de AV. Nuestro modelo geométrico proporciona los valores críticos por debajo de los cuales este índice sugeriría que las malformaciones del aparato subvalvular mitral deben abordarse si se considera la estrategia de reparación biventricular.

Hasta el momento, no se ha podido identificar un conjunto de medidas con puntos de corte precisos que permitan una toma de decisiones quirúrgicas confiable. Sumado a esta limitación, sabemos que esta patología puede presentar disbalance a diferentes niveles (auricular, valvular, subvalvular, ventricular y arterial) y verse influenciada por el interjuego de los lechos vasculares sistémico y pulmonar ${ }^{(8)}$. Es por esto que su abordaje es complejo y deben evaluarse anatómica y hemodinámicamente en forma completa y posiblemente con una modalidad multi-imagen, especificando siempre el nivel anatómico afectado (Figura 3).

A pesar de muchas incertidumbres, la mejora en el entendimiento de la enfermedad y la introducción y aplicación de nuevos indicadores ecocardiográficos 
han proporcionado una herramienta útil para la toma de decisiones quirúrgicas en esta población (Figura 4).

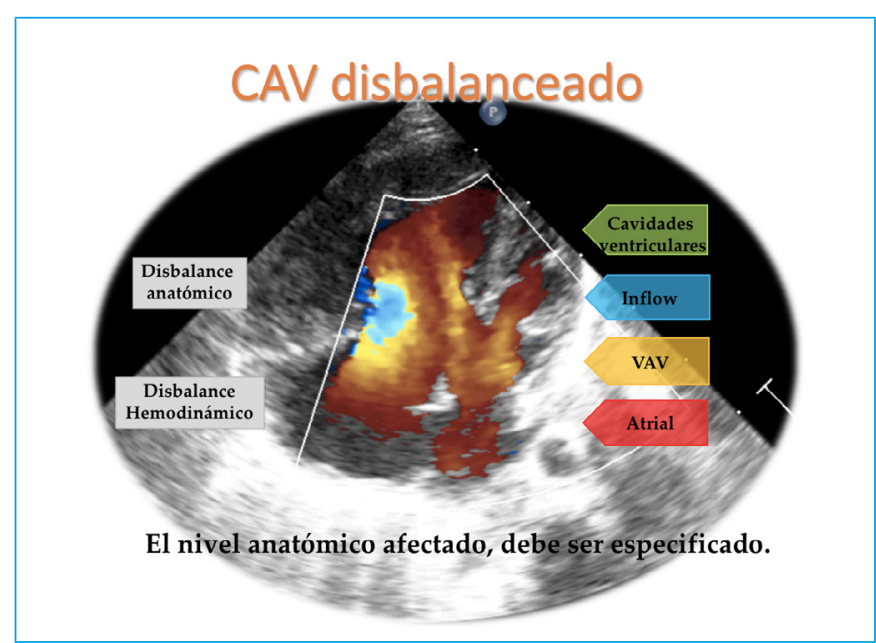

Figura 3. Disbalance anatómico y hemodinámico en el canal atrioventricular disbalanceado. CAV: canal atrio-ventricular

\section{Ideas para recordar}

- El balanceo el canal atrio-ventricular un nivel, no implica que lo sea en los otros.

- El nivel anatómico afectado debe ser especificado.

- Con la obtención de una vista 4 cámaras apical blanco y negro y con Doppler color, se pueden obtener múltiples mediciones que facilitan la toma de decisiones quirúrgicas.

\section{Bibliografía}

1. Cohen M, Marshall J, Paul M, Weinberg P, et al. Morphometric Analysis of Unbalanced Common Atrioventricular Canal Using Two-Dimensional Echocardiography. J Am Coll Cardiol 1996;28:1017.

2. Jegatheeswaran A, Pizarro C, Caldarone C, et al. Echocardiographic Definition and Surgical Decision-Making in Unbalanced Atrioventricular Septal Defect. Circulation 2010;122[suppl 1]:S209-S215.

3. Lugones I, Biancolini F, Biancolini J, et al. Feasibility of Biventricular Repair in Right Dominant Unbalanced Atrioventricular Septal Defect: A New Echocardiographic Metric to Refine Surgical Decision-Making. World Journal for Pediatric and Congenital Heart Surgery 2017;8(4):460-467.

4. Nathan M, Liu H, Pigula F, et al. Biventricular Conversion After Single-Ventricle Palliation in Unbalanced Atrioventricular Canal Defects. Ann Thorac Surg 2013;95:2086-96

5. Cohen M, Jacobs M, Weinberg P, et al. Morphometric Analysis of Unbalanced Common Atrioventricular Canal Using Two-Dimensional Echocardiography. J Am Coll Cardiol 1996;28:1017-23.

6. Szwast A, Marino B, Rychik J, et al. Usefulness of Left Ventricular Inflow Index to Predict Successful Biventricular Repair in Right-Dominant Unbalanced Atrioventricular Canal. Am J Cardiol 2011;107:103-109.

7. Meza J, Devlin P, Overman D, et al. The CHSS Complete Atrioventricular Septal Defect Cohort: Baseline, Pre-Intervention Echocardiographic Characteristics. Seminars in Thoracic and Cardiovascular Surgery 2018; 31(1):80-86

8. Lugones I, Biancolini F, Lugones G, et al. The matter of "unbalance" in right dominant atrioventricular septal defect. Ann Pediatr Card 2019;12:132-4.

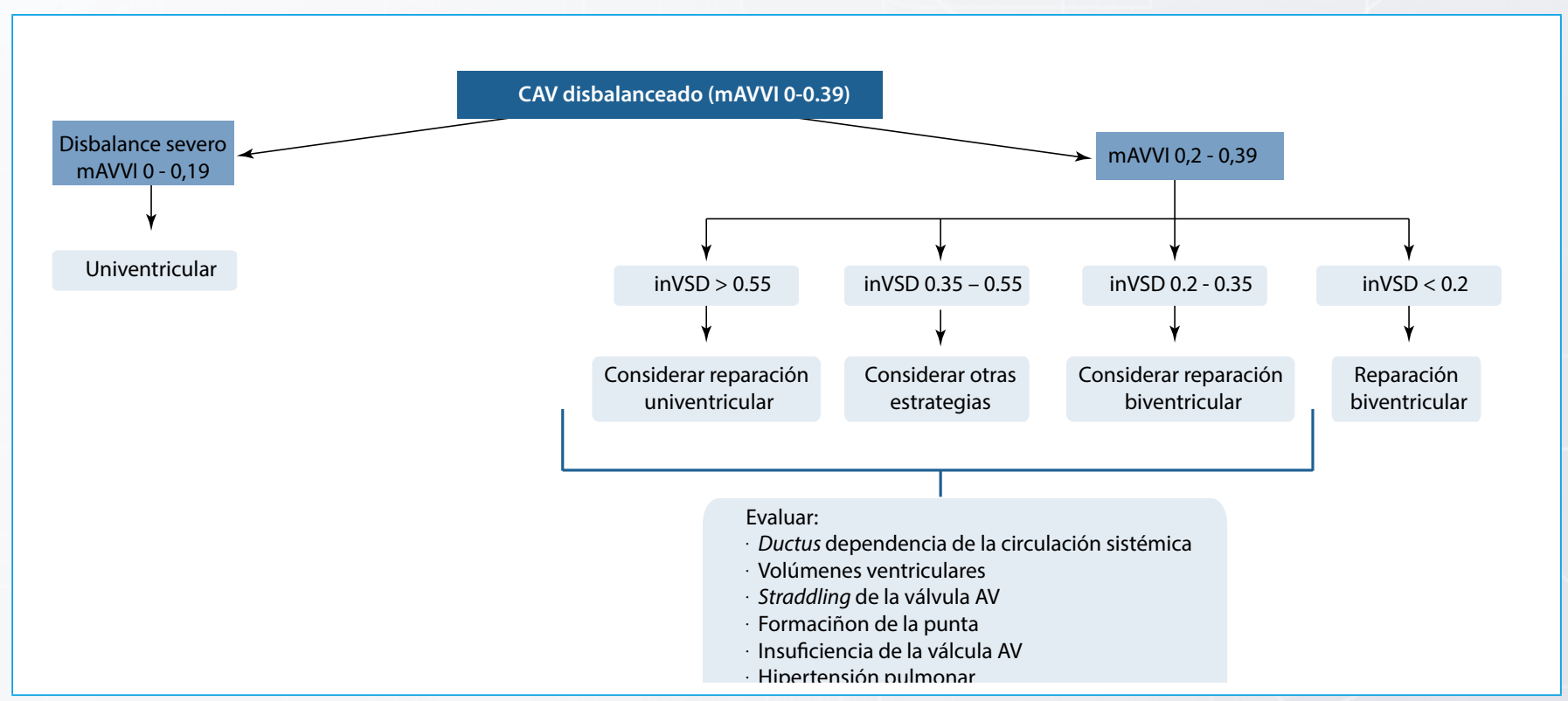

Figura 4. Algoritmo sugerido para la toma de decisiones quirúrgica en pacientes con canal atrio-ventricular disbalanceado dominante derecho 